\title{
Happy birthday, Genetics in Medicine!
}

\author{
James P. Evans, MD, PhD ${ }^{1}$
}

As we publish the January 2018 issue of Genetics in Medicine (GIM), we celebrate this journal's twentieth anniversary. When GIM was conceived by its first editor-in-chief, Richard King, and published (as a bimonthly journal) in November 1998, the world was a different place. In the broader societal context, Bill Clinton was president, the Dow Jones Industrial Average closed the year at 9,181, a search engine called "Google" was founded, and Barack Obama was a little-known state senator in Illinois. The world of genetics was a very different place; indeed, the very name of the College was different, with "Genomics" absent from its title. 1998 saw publication of the genome of C. elegans ${ }^{1}$ but it would be two more years until Drosophila melanogaster's genome would be sequenced. ${ }^{2}$ And while the Human Genome Project had been chugging along since its launch in 1990, we were still a year away from decoding even the first human chromosome (chromosome 22$)^{3}$ and five years away from elucidating the sequence of the rest of our own species' genome. ${ }^{4}$

Perusal through back issues of Genetics in Medicine shows us just how profoundly our field has changed. Genes were being analyzed laboriously, one at a time via Sanger sequencing; chromosomal microarrays were nowhere to be seen in the pages of the journal; and, of course, the notion of "whole-genome sequencing" individual patients in a clinical context was the stuff of science fiction. Through the years GIM has been fortunate to publish important "firsts" in our field, including the first full clinical report of the use of wholegenome sequencing to diagnose and guide treatment of a patient ${ }^{5}$ and the first use of noninvasive prenatal screening via circulating placental DNA in the maternal circulation to screen for fetal trisomies 21,18 , and $13 .{ }^{6}$ Our pages are now full of technological wonders it would have been hard to imagine in 1998.

But it's vital to also note that some things haven't changed. The importance of careful clinical phenotyping and a good family history, for instance, have never been more critical than now as we struggle with interpreting untold numbers of variants found in our patients. Moreover our field, and thus GIM, continues to place the broader, social significance of technological and medical advances in perspective, grappling with thorny issues that transcend science and ultimately rely on value judgments and thoughtful consideration of complex
- and sometimes controversial-topics. And just as has been the case these past 20 years, one of the most vital functions of GIM will be to continue the dissemination of important recommendations from the American College of Medical Genetics and Genomics through practice guidelines, points to consider, and clinical recommendations that have been developed through careful consideration of evidence and thoughtful deliberation.

To help celebrate Genetics in Medicine's twentieth birthday, we are featuring a series of invited review articles that will explore significant areas of progress in our field. These invited reviews will be authored by luminaries who have graciously agreed to address some of the most critical current topics in medical genetics and genomics. Upcoming reviews, which will run every few months throughout 2018 and beyond, will start with Muin Khoury and colleagues' exploration of the past, present, and future of genomics in the realm of public health. We will also feature GIM editor Maren Scheuner's examination of how best to apply principles of evidence-based medicine to genomics, a topic that is particularly timely as our field is subjected to tremendous pressures to expand implementation in the face of limited evidence. James Lupski and colleagues will explore the future of neurogenetics, and Nicola Longo will report on continued advances in an iconic genetic disorder, phenylketonuria. Jonathan Berg and colleagues will provide a timely overview of variant interpretation and address "big questions" in this landscape, including how variant interpretation is entangled with notions of penetrance and how accurate adjudication can be scaled to present and future needs. Steven Scherer and colleagues will review the explosion of recognized recurrent microdeletion/duplication syndromes, and Hutton Kearney, of GIM's editorial board, will discuss displacement of the traditional karyotype in clinical cytogenetics with prospects for the future. Highlighting the considerable expansion of genomics to other medical fields, we will also feature twentieth-anniversary reviews that focus on the burgeoning use of genomic analysis in oncology as well as the revolution that genomics has ushered into obstetrics and gynecology; the latter to be authored by Tony Gregg, the president-elect of ACMG. Likewise, Katrina Goddard will review developments in carrier screening for reproductive decision making, and we

${ }^{1}$ Center for Genomics and Society, University of North Carolina at Chapel Hill, Chapel Hill, North Carolina, USA; ${ }^{2}$ Department of Genetics, University of North Carolina at Chapel Hill, Chapel Hill, North Carolina, USA; ${ }^{3}$ Department of Medicine, University of North Carolina at Chapel Hill, Chapel Hill, North Carolina, USA. Correspondence: James P. Evans (jpevans@med.unc.edu)

Submitted 16 October 2017; accepted 16 October 2017; advance online publication 7 December 2017. doi:10.1038/gim.2017.205 
will explore the dramatically changing landscape of genetic counseling, something that affects every aspect of medical genetics and beyond.

GIM is also committed to continuing its leadership as our field struggles with how (and sometimes whether) to apply technological advances that can challenge fundamental assumptions and underpinnings of a just society. Thus, Erick Parens will discuss the ethical implications of emergent changes in prenatal screening, M.K. Thong will explore the development of genomics in emerging countries and its relevance to women's reproductive rights, Mark Rothstein will review legal challenges that face our field, and Paul Lombardo will explore how past and current perceptions of eugenics inform current and future ethical debates. Finally, with a nod to the primacy of technological development as a driver of our field, Wayne Grody, a past president of ACMG, will provide a review that looks forward to emerging genetic and genomic technologies. All of these reviews will be accompanied by social media campaigns, something that didn't exist 20 years ago but have now become central to the dissemination of the College's messages and the research published in GIM's pages (online as well as physical-for now). What would we have thought 20 years ago, with Twitter almost a decade in the future, had we been told that one way GIM would be getting its messages across in 2018 would be through "tweeting"?!

As we enter GIM's third decade, we can be proud of our field. The medical genetics community has led the charge in applying wondrous technologies to reduce the human suffering that results from the vagaries of human genetic variation. Let us proceed into the future with the hope that, while the past decade will be remembered as the decade of improved diagnostics, the next will see accelerating therapeutic successes that materially benefit those who suffer from genetic disorders. We live in exciting times. But we also must be on our guard. Our own understandable enthusiasm, as well as well as the equally understandable excitement on the part of the average citizen about the potential of genetics, has led to a great deal of commercial activity. While responsible and evidence-based commercial endeavors can be a boon for both the public and providers, we unfortunately see shameless promotion of ludicrous products and evidence-free claimsthe dogged persistence of which prove that there's a sucker born every minute and that, alas, there will always be those who will be seduced into peddling snake oil instead of engaging in honest assessment of the evidence.

Over the next two decades, we must make evidence of meaningful clinical benefit our lodestar. And we as a community must be the ones to not only generate the necessary evidence but also insist on its primacy over unbridled enthusiasm, wishful thinking, and greed. In all of this, Genetics in Medicine will endeavor to be a faithful partner. The future is bright...onward and upward!

\section{DISCLOSURE}

The author declares no conflict of interest.

\section{REFERENCES}

1. Genome sequence of the nematode C. elegans: a platform for investigating biology. Science 1998;282:2012-2018.

2. Myers EW, Sutton GG, Delcher $A L$, et al. A whole-genome assembly of drosophila. Science 2000;287:2196-2204.

3. Dunham I, Hunt AR, Collins JE, et al. The DNA sequence of human chromosome 22. Nature 1999;402:489-495.

4. International Human Genome Sequencing Consortium. Finishing the euchromatic sequence of the human genome. Nature 2004;431: 931-945.

5. Worthey EA, Mayer AN, Syverson GD, et al. Making a definitive diagnosis: successful clinical application of whole exome sequencing in a child with intractable inflammatory bowel disease. Genet Med 2011;13:255-262.

6. Palomaki GE, Deciu C, Kloza EM, et al. DNA sequencing of maternal plasma reliably identifies trisomy 18 and trisomy 13 as well as Down syndrome: an international collaborative study. Genet Med 2012;14: 296-305. 